\title{
Proton magnetic resonance spectroscopy in the evaluation of patients with acute Charcot neuro-osteoarthropathy
}

\author{
Francesca Bolacchi • Luigi Uccioli • Salvatore Masala • Laura Giurato • \\ Valeria Ruotolo • Marco Meloni • Eleonora Baffari • Elenia Cinelli • \\ Marcello Cadioli • Ettore Squillaci • Giovanni Simonetti • Alberto Bergamini
}

Received: 7 October 2012 /Revised: 31 March 2013 /Accepted: 3 April 2013 /Published online: 11 June 2013

(C) European Society of Radiology 2013

\begin{abstract}
Objective To evaluate whether bone marrow proton magnetic resonance spectroscopy (MRS) might provide a quantitative parameter able to assess disease activity in acute Charcot neuro-osteoarthropathy $(\mathrm{CN})$.

Methods Ten diabetic patients with stage $0 \mathrm{CN}$ were prospectively evaluated at clinical onset and during treatment follow-up. The MRS lipid spectrum was analysed and a lipid polyunsaturation index (PUI) was calculated. Disease recovery was defined as the disappearance of bone marrow oedema as demonstrated on MRI short-tau-inversion-recovery (STIR) images. A 3-T MRI was used.

Results Inter- and intra-individual PUI measurements generated reproducible results with approximately $7 \%$ and $6 \%$ variation respectively. Baseline PUI values were significantly higher in patients with acute $\mathrm{CN}$ compared with controls. Also, a significant positive correlation was observed between baseline PUI values and serum levels of IL- 6 and TNF- $\alpha$. During follow-up a gradual decrease in PUI was observed. The percentage reduction of PUI values at 3 months'
\end{abstract}

F. Bolacchi $(\bowtie) \cdot$ S. Masala $\cdot$ E. Squillaci $\cdot$ G. Simonetti Department of Diagnostic Imaging, Molecular Imaging, Interventional Radiology, and Radiation Therapy, University of Rome "Tor Vergata", Viale Oxford 81,

00133 Rome, Italy

e-mail: f.bolacchi@tiscali.it

L. Uccioli $\cdot$ L. Giurato $\cdot$ V. Ruotolo $\cdot$ M. Meloni

Department of Internal Medicine, University of Rome

"Tor Vergata", Rome, Italy

E. Baffari $\cdot$ E. Cinelli $\cdot$ A. Bergamini

Department of Public Health and Cellular Biology, University of Rome "Tor Vergata", Rome, Italy

M. Cadioli

Philips Medical Systems, Milan, Italy follow-up with respect to baseline values showed a negative correlation with recovery time.

Conclusions Bone marrow MRS may provide a measurable index that allows progressive evaluation of disease activity in acute CN. MRS may be a complementary tool that can be used to guide clinicians in the management of acute $\mathrm{CN}$ patients.

Key Points

- Bone marrow MRS demonstrates lipid alterations in acute Charcot neuro-osteoarthropathy (CN).

- Bone marrow MRS allows disease activity in acute CN to be evaluated.

- MRS could become a new tool in the management of CN.

Keywords Charcot neuro-osteoarthropathy · Magnetic resonance spectroscopy $\cdot$ Bone marrow $\cdot$ Lipids $\cdot$ Inflammation

\section{Introduction}

Charcot neuroarthropathy $(\mathrm{CN})$ occurs most commonly in the foot/ankle in diabetic patients with sensory neuropathy and is a common cause of morbidity in this population. In its early stage $\mathrm{CN}$ is characterised by acute inflammation, which may subsequently lead to chronic bone alterations such as bone and joint fracture, dislocation, instability and gross deformities [1-3]. Painlessness and abnormal foot biomechanics play an important part in the pathogenesis of the disorder. However, recent attention has focussed on several abnormalities, which together suggest a more complex cause. In particular, several authors have drawn attention to the possible link between proinflammatory cytokines and neuroarthropathy in the context of an exaggerated inflammatory response to trauma $[4,5]$. The mainstay of the initial management of acute $\mathrm{CN}$ is immobilisation and offloading in a plaster cast. Strict and careful treatment follow-up is of pivotal importance in order to prevent the 
development of the devastating bone and joint alterations associated with the late stages of this disease [6, 7]. However, in the acute stage of $\mathrm{CN}$ there are no laboratory criteria and no haematological markers to assess disease activity [8]. Therefore, up to now, the follow-up of stage $0 \mathrm{CN}$ patients has been based on local clinical signs (erythema, swelling and skin temperature) [7, 9]. Although local clinical signs are useful indicators of the outcome of the disease, they are affected by poor sensibility and reproducibility $[10,11]$. Hence, a reliable and objective technique would be highly desirable to assess the disease course. Ideally, this technique should be able to measure the biochemical changes associated with the inflammatory process associated with acute $\mathrm{CN}$.

Proton MR spectroscopy (MRS) is a MR technique that has recently been increasingly used in clinical practice. MRS gives information about the chemical/metabolic composition of the tissue, providing a spectrum of the intensity of the signal emitted from different tissue metabolites. In particular, MRS can detect reliably different compartments of lipids [12-15]. Of particular interest are indices that provide measures of the unsaturation levels among the triglycerides, which have proved useful in the quantitative assessment of bone marrow inflammatory lesions [16]. Based on these premises, we undertook an in vivo study to evaluate the possible presence of bone marrow lipid alterations by proton MRS in acute $\mathrm{CN}$ patients that could be used to assess and monitor disease activity.

\section{Materials and methods}

Patients and controls Ten diabetic patients (four type 1, six type 2 diabetes) with acute $\mathrm{CN}$ were prospectively enrolled in the study. Acute CN was defined based on the following clinical and MRI signs: unexplained, relatively painless, increasing swelling of a foot and ankle, skin temperature increase of at least $2{ }^{\circ} \mathrm{C}$ compared with the contralateral foot $(\Delta \mathrm{T})$, clinical instability due to ligamentous injury/occult trauma and presence of typical bone marrow oedema on MRI. All patients were in stage 0 according to the Eichenholtz stage system and were free of any structural bone or articular alteration, as documented by computed tomographic evaluation, active foot ulceration and/or signs of soft tissue infection. All patients had unilateral foot involvement as documented by MRI (no evidence of bone marrow oedema) as well as clinical evaluation. All patients displayed severe peripheral sensory polyneuropathy. The presence of peripheral neuropathy was assessed by the vibration perception threshold (VPT expressed in volts) and the diabetic neuropathy index (DNI). Peripheral neuropathy was defined by a VPT $\geq 25$ volts and/or a positive DNI score $>2$ points [17]. Acute Charcot MRS baseline evaluation of the affected foot and contralateral foot was performed within $24 \mathrm{~h}$ of meeting enrolment criteria. Following the diagnosis of acute $\mathrm{CN}$, all patients underwent foot offloading and immobilisation by serial total contact casting with progression to removable cast walkers. Follow-up included clinical, MRI and MRS evaluation of the affected foot every 4 weeks. Recovery was defined by the disappearance of bone marrow oedema as demonstrated on MRI using T2-weighted short tau inversion recovery (STIR) images. Also, proinflammatory cytokine (IL-6 and TNF- $\alpha$ ) serum concentrations were analysed in acute $\mathrm{CN}$ patients. For the evaluation of the serum levels of interleukin-6 (IL-6) and tumour necrosis factor $\alpha$ (TNF- $\alpha$ ) blood samples were obtained by peripheral venipuncture within $24 \mathrm{~h}$ of meeting the enrolment criteria. Samples were processed as described elsewhere [4]. A group $(n=10)$ of ageand sex-matched diabetic patients with peripheral sensory polyneuropathy, without clinical and radiological evidence of a history of $\mathrm{CN}$, was also studied. Healthy sex- and age( \pm 5 years) matched controls $(n=10)$ with no evidence of diabetes mellitus (according to self-reported absence of antidiabetic medication) and a comparable body mass index $\left( \pm 1 \mathrm{~kg} / \mathrm{m}^{2}\right)$ were also included. This study was conducted according to the principles expressed in the Declaration of Helsinki and approved by our institutional review board. Informed consent was obtained from all subjects before the performance of the study.

MR Examinations MRS and MRI were performed at $3 \mathrm{~T}$ (Achieva, Philips Medical Systems, Best, The Netherlands). All imaging and spectra were acquired and analysed in consensus by two radiologists experienced with MRS and with musculoskeletal imaging. The radiologists were fully blinded to the clinical patient data and to the results of previous or current diagnostic imaging techniques. Subjects were examined in the supine position, placing the feet first into the gantry. All examinations were performed with a quadrature-type birdcage (QD) coil. Presence or absence of bone marrow oedema within different bones of the foot and ankle was determined using T2-weighted short tau inversion recovery (STIR) images acquired in the coronal, axial and sagittal planes.

Spectroscopic data were obtained using a single-voxel point-resolved spectroscopic sequence (PRESS) as previously described [14] (TR/TE 3,000/37, spectral BW of $5 \mathrm{kHz}, 64$ averages, 4,096 data points). The PRESS sequence was water suppressed using a selective excitation pulse to crush the water signal. The voxel (volume of interest, VOI) size was $1.3 \times 1.3 \times 1.3 \mathrm{~cm}^{3}$. The VOI was placed within the largest bone presenting bone marrow oedema (Fig. 1). Metatarsal bones were not taken into account in order to exclude signal heterogeneities at the distal end of the field of view. Fully automated frequency determination, power optimisation and shimming phases 


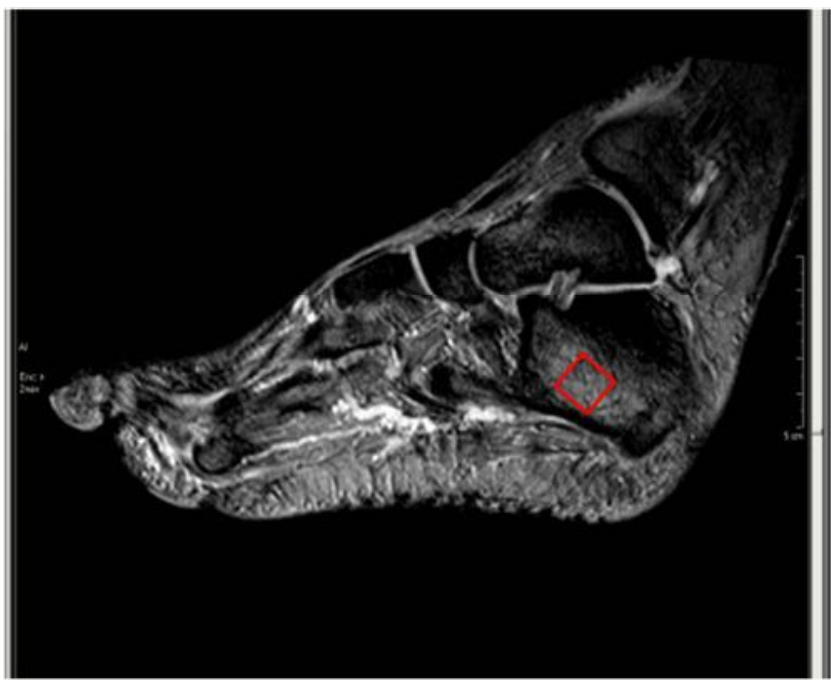

Fig. 1 A 57-year-old woman with $\mathrm{CN}$ acquired in the affected foot at baseline. MR image shows voxel positioning

were performed in the VOI. The shimming was performed using a first order shimming protocol. To confirm the reproducibility of the measurements, three sets of spectral acquisition were repeated three times each in four patients and six control subjects.

Processing of Spectral Data The spectrum was inspected, and the signal-to-noise ratio of each peak included in the polyunsaturation index (PUI) calculation was measured. When the ratio for each peak was $>2$, the spectrum was analysed. Data were analysed using the jMRUI v4.0 software package [18]. Zero and first-order phase correction was estimated by AMARES [19]. Soft constraints were used during the fitting procedures. The maximal metabolite line width was limited to $25 \mathrm{~Hz}$, and peak shifts were restricted to $\pm 0.05 \mathrm{ppm}$ of the theoretical peak location to allow some flexibility to the fitting algorithm. A Lorentzian model function was assumed for each peak. Metabolites to be estimated were defined with a reference database of known peaks [20]. The metabolite peaks were assigned as follows: olefinic protons $(-\mathrm{CH}=\mathrm{CH}-), 5.35 \mathrm{ppm}$; diallylic protons $(=\mathrm{CH}-$ $\left.\mathrm{CH}_{2}-\mathrm{CH}=\right), 2.8 \mathrm{ppm}$; allylic protons $(-\mathrm{CH} 2-\mathrm{CH}=\mathrm{CH}-)$, 2.03; bulk methylene protons $\left(-\mathrm{CH}_{2}-\right)_{\mathrm{n}}, 1.3 \mathrm{ppm}$; terminal methyl protons $\left(-\mathrm{CH}_{3}\right), 0.9 \mathrm{ppm}$. The spectra acquired were analysed using the AMARES algorithm, in which the differences between the acquired spectra are iteratively minimised and the model-based spectrum are defined a priori on the basis of the database and the Lorentzian constraint. After the algorithm had been applied, the script displayed the estimated peak areas. The metabolite ratio index of polyunsaturation (PUI) was defined as the signal amplitude of the diallylic fatty acid functional group resonance divided by the sum of the allylic, diallylic, bulk methylene and terminal methyl functional group resonances: $\mathrm{PUI}=$
$\mathrm{I}_{\text {diallylic }}\left(\mathrm{I}_{\text {allylic }}+\mathrm{I}_{\text {diallylic }}+\mathrm{I}_{\text {bulk methylene }}+\mathrm{I}_{\text {terminal methyl }}\right)$ [21]. Evaluation of Cramer-Rao lower bounds was performed for each metabolite of the fitted spectra using LCModel software [22]. We required the metabolite Cramer-Rao value to be $<$ $15 \%$ for inclusion in data analyses.

Statistical Analysis The Mann-Whitney U test was used for two-group comparisons. One-way ANOVA test with Dunnett's multiple comparison test was used for multiple group comparisons. Correlations were performed by Spearman rank correlation coefficient. Intraindividual and interindividual variations were assessed using the coefficient of variation $(\mathrm{CV})$. All $P$ values are two-tailed. $P$ values $<0.05$ were considered significant. Statistical analyses were performed using the SPSS (SPSS Inc., Version 10.0, Chicago, IL, USA) statistical package.

\section{Results}

Patients The mean age was similar in the acute $\mathrm{CN}$ subjects (male/female: $5 / 5$, age $58 \pm 10.3$ ), diabetic control subjects (male/female: 6/4, age $64 \pm 12.9$ ) and healthy control participants (male/female: $4 / 6$, age $51 \pm 7.4$ ). The mean duration of diabetes (years, mean $\pm \mathrm{SD}$ ) was $20.6 \pm 9.7$ in acute $\mathrm{CN}$ subjects and $21.4 \pm 10.5$ in diabetic control subjects. The percentage $( \pm \mathrm{SD})$ of Hbalc was $8.3 \pm 2$ in acute $\mathrm{CN}$ case subjects and $8.8 \pm 1.9$ in diabetic control subjects. All diabetic patients were on insulin therapy. Lesions were classified according to the site: hindfoot lesions involved the tibia, fibula, calcaneus or talus, midfoot lesions involved the cuneiforms, navicular or cuboid, and forefoot lesions involved the metatarsals or phalanges (when multiple sites were affected, classification was based on the most proximally affected bone). The lesion sites were hindfoot, $n=6$, and midfoot, $n=4$. Mean healing time on MRI was $9.23 \pm$ 2.1 months.

MRS evaluation of disease activity at baseline Repeated inter- and intra-individual PUI measurements generated reproducible results with approximately $7 \%$ and $6 \%$ variation respectively. At baseline, PUI values measured in acute $\mathrm{CN}$ case subjects $(0.054 \pm 0.007)$ were significantly higher than in the contralateral foot of acute $\mathrm{CN}$ patients $(0.023 \pm 0.0052, P<0.001)$, diabetic control subjects $(0.026 \pm 0.0051, P<0.001)$ and healthy controls $(0.026 \pm 0.005, P<0.001)$ (Fig 2). As also shown in Fig. 2, PUI values were not different in controls $(P>0.05)$. As summarised in Table 1 , baseline serum concentrations of IL-6 and TNF- $\alpha$ were higher in acute $\mathrm{CN}$ patients than in controls and showed a 


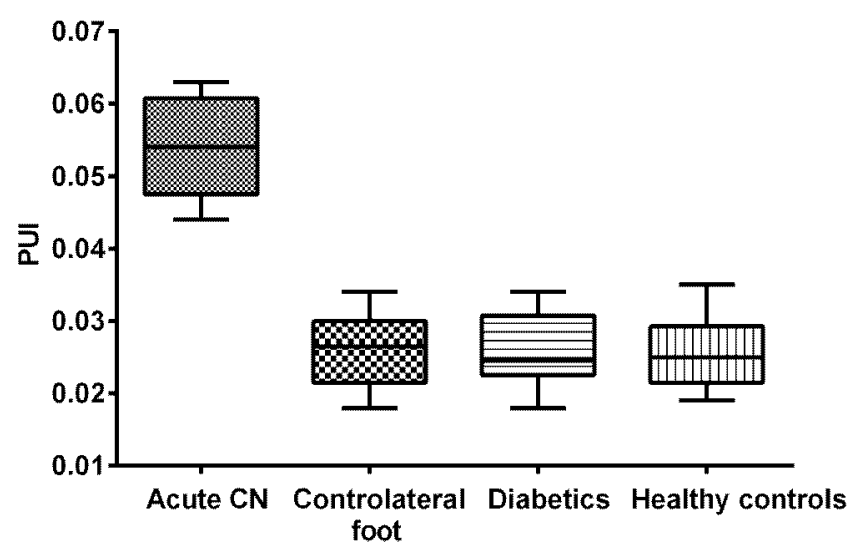

Fig. 2 Polyunsaturation index (PUI) measurement in acute Charcot neuro-osteoarthropathy $(\mathrm{CN})$ patients and controls. Baseline MRS was performed within $24 \mathrm{~h}$ of the diagnosis of acute $\mathrm{CN}$. The voxel was placed within the bone of the largest dimension of the affected foot showing bone oedema on MRI (calcaneus, $n=6$; cuboid, $n=4$ ); the same bones were studied in the contralateral foot of patients, diabetic subjects and healthy controls

significant positive correlation with PUI values in acute CN patients.

MRS evaluation of disease activity during follow-up Figure 3 shows data on the MRS evaluation of a single patient basis during follow-up. As the PUI mean \pm SD value of the controls was $0.023 \pm 0.004$, a cutoff value of $0.023+2 \mathrm{SD}$ was arbitrarily selected. Consequently, the decrease in PUI during follow-up was considered significant if PUI reached a value of at least 0.031 . The mean time of PUI normalisation was $8.4 \pm$ 1.9 months. A gradual reduction of PUI was observed during follow-up. Mean \pm SD PUI values during followup were as follows: baseline, $0.054 \pm 0.007 ; 3$ months, $0.044 \pm 0.0075 ; 6$ months, $0.037 \pm 0.0087$; recovery, $0.025 \pm 0.0033$. Interestingly, the percentage reduction of PUI at 3 months' follow-up with respect to baseline values showed a negative correlation with recovery time as assessed by bone marrow oedema disappearance $(r=-0.756,95 \% \mathrm{CI}=-0.938--0.241, P-0.011$; Fig. 4). Figure 5 shows examples of marrow spectra

Table 1 Correlation between baseline PUI values of the affected foot in acute $\mathrm{CN}$ patients and serum IL- 6 and TNF- $\alpha$ concentrations

\begin{tabular}{llll}
\hline & $\begin{array}{l}\text { Baseline cytokine } \\
\text { concentration }(\mathrm{pg} / \mathrm{ml})\end{array}$ & PUI & \\
\cline { 3 - 4 } & & $r(95 \% \mathrm{CI})$ & $P$ value \\
\hline IL-6 & $12.9 \pm 7.3^{*}$ & $0.705(0.135-0.924)$ & 0.023 \\
$\mathrm{TNF}-\alpha$ & $5.6 \pm 3.5^{*}$ & $0.747(0.222-0.936)$ & 0.013 \\
\hline
\end{tabular}

*Mean \pm SD. Serum cytokine concentrations in diabetic control subjects and healthy controls were as follows: IL-6, 5.1 \pm 2.8 and $4.9 \pm 1.9$ $(P<0.001$ with respect to $\mathrm{CN}) ; \mathrm{TNF}-\alpha, 1.8 \pm 0.8$ and $2.3 \pm 1.6(P<0.001$ with respect to $\mathrm{CN})$, respectively

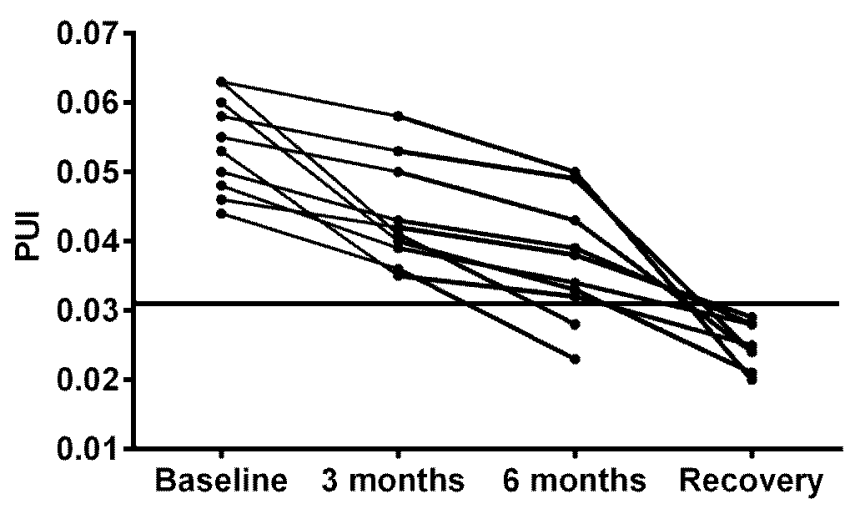

Fig. 3 PUI variations during follow-up. PUI evaluation on a single patient basis during follow-up. Follow-up MRIs were performed every 4 weeks. Spectra were re-evaluated placing the VOI at the same level as at the baseline examination

acquired from typical $\mathrm{CN}$ patients at baseline, during follow-up and from control subjects.

\section{Discussion}

In this study, patients with acute $\mathrm{CN}$ were prospectively evaluated by MRS of the bone marrow. The lipid spectrum was analysed and the level of lipid unsaturation was evaluated by the calculation of a polyunsaturation index (PUI). Our findings indicate that PUI is an MRS parameter that has good reproducibility and that is able to discriminate acute $\mathrm{CN}$ patients from controls. In addition, after limb immobilisation, a progressive reduction of PUI was observed in all patients. Finally, the alterations of PUI found in the acute CN patients were not constitutive, as PUI values obtained in the

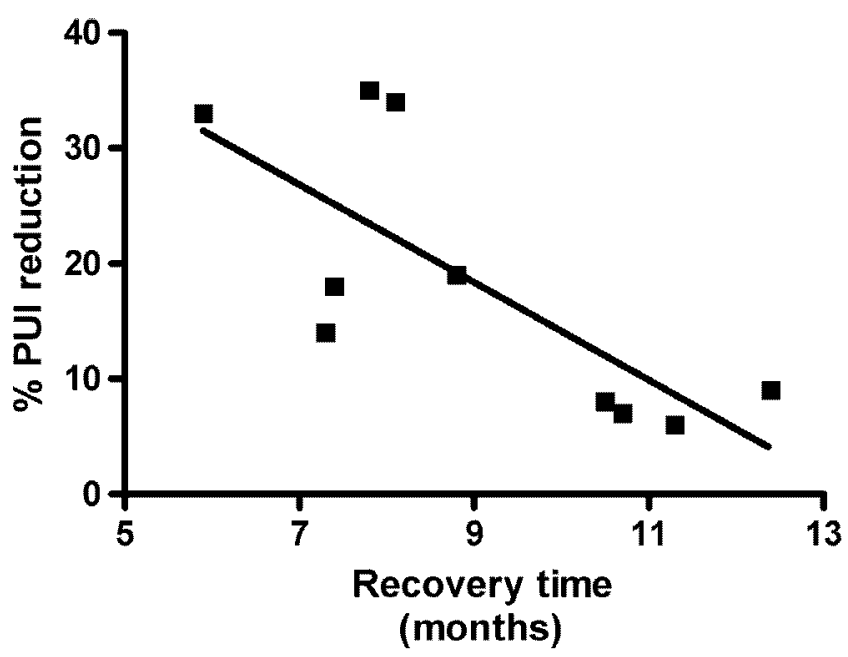

Fig. 4 Correlation of the percentage reduction of PUI values at 3 months' follow-up with recovery time as assessed by MRI. The percentage reduction of PUI values at 3 months' follow-up was calculated with respect to baseline values. Healing was defined by the absence of bone marrow oedema on MRI 
Fig. 5 MRS spectra of a $\mathrm{CN}$ patient and of control subjects. Lipid spectrum from a 61-yearold woman with $\mathrm{CN}$ acquired in the affected foot at baseline (a), at 3 months' follow-up (b) and at MRI with evidence of healing, i.e. absence of bone marrow oedema (c). Spectrum acquired in the contralateral (unaffected) foot of the same patient at baseline (d).

Spectrum acquired in the foot of a diabetic control (e) and in the foot of a healthy control (f). Arrows: $1=$ diallylic protons (=CH-CH2-CH=), 2.8 ppm; $2=$ allylic protons $(-\mathrm{CH} 2-\mathrm{CH}=$ $\mathrm{CH}-), 2.03$ ppm; 3=bulk methylene protons $\left(-\mathrm{CH}_{2}-\right)_{\mathrm{n}}$, $1.3 \mathrm{ppm} ; 4=$ terminal methyl protons $\left(-\mathrm{CH}_{3}\right), 0.9 \mathrm{ppm}$
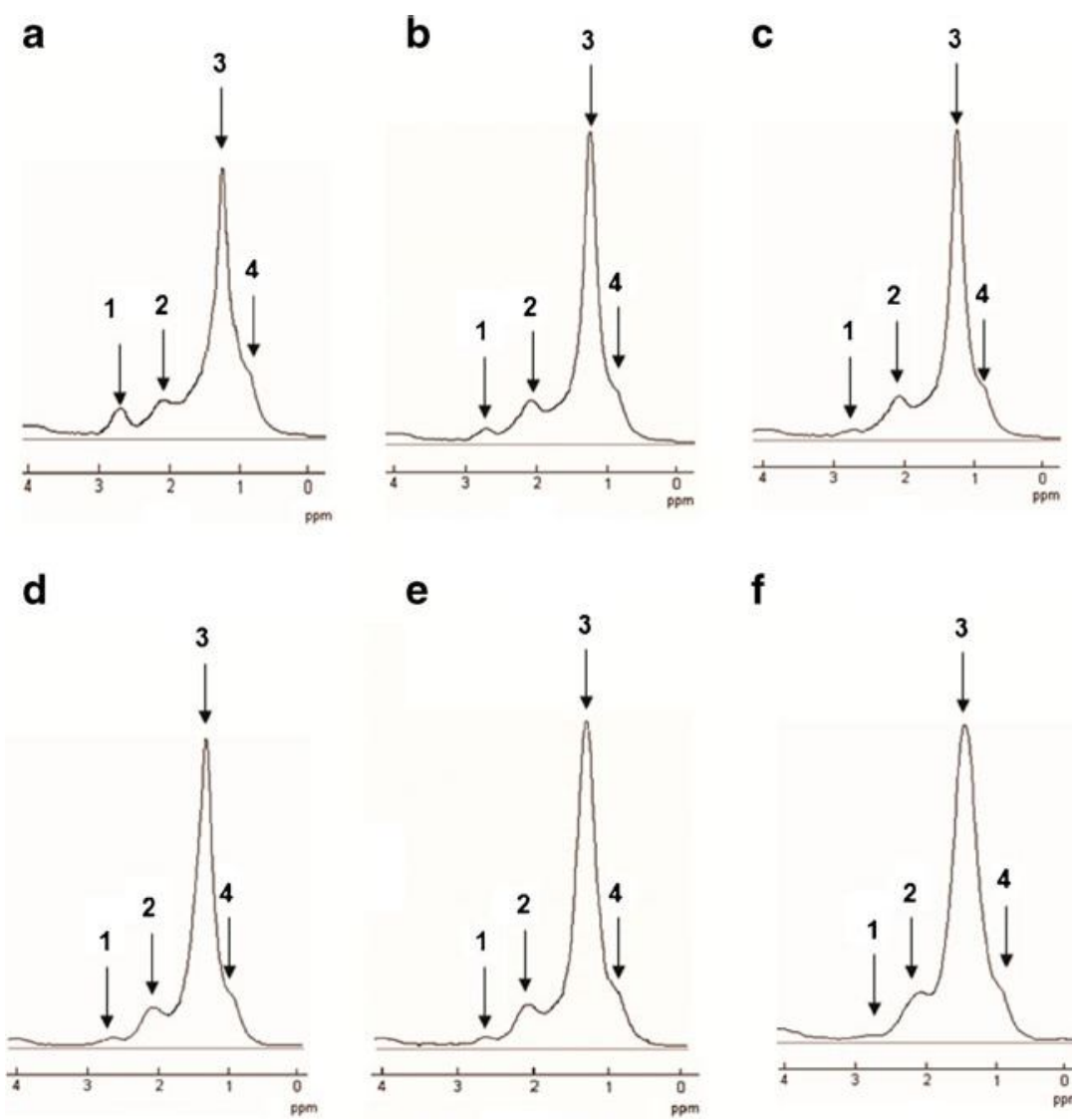

e

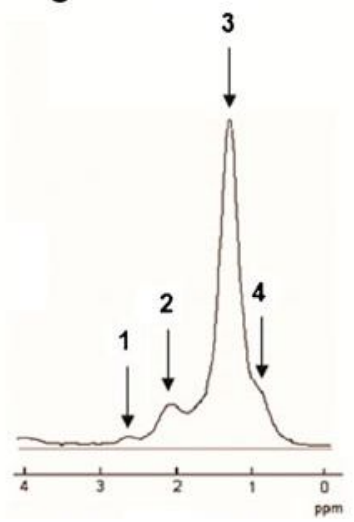

f

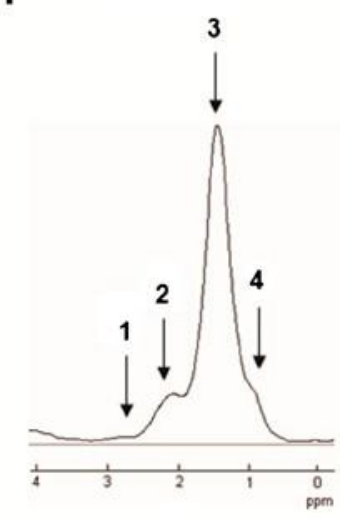

contralateral foot of these subjects were not different from those found in control subjects.

$\mathrm{Up}$ to now the evaluation of acute $\mathrm{CN}$ patients has relied on local inflammatory signs such as skin temperature, swelling and erythema [7-9]. Local clinical signs are useful to guide the physician in shifting from the cast to other pressure-relieving devices. However, they are affected by poor specificity and reproducibility and their utility in providing a measure of disease activity level has been recently questioned $[10,11]$. The lack of specific criteria and objective indices able to assess the disease activity during follow-up, in addition to being a hindrance to the implementation of current physical therapeutic options, has also limited research concerning pharmacological treatments for acute $\mathrm{CN}$. Recently, MRI has been introduced in the evaluation of acute $\mathrm{CN}$. In particular, the disappearance of bone marrow oedema as evaluated on STIR images has proved useful in assessing disease recovery [23]. However, during the period in between disease onset (presence of bone marrow oedema) and recovery (absence of bone marrow oedema) the regression of bone marrow oedema is difficult to quantify [24]. The use of contrast-enhanced MRI has been advocated [24]. However, repeated contrastenhanced examinations are not always feasible in diabetic patients [25]. In this clinical contest, a quantitative noninvasive index would be highly desirable to progressively monitor disease activity. Over the last decade various pathophysiological hypotheses have been suggested for acute $\mathrm{CN}$. Recently, micro-trauma has been proposed to be the initial trigger of the uncontrolled inflammatory response that characterises acute $\mathrm{CN}[4,5]$. Generally, the inflammatory response involves several pathophysiological changes such as the production of proinflammatory cytokines. In our study we found that the serum levels of the proinflammatory cytokines IL- 6 and TNF- $\alpha$ were significantly increased at baseline in acute $\mathrm{CN}$ patients compared to controls. This is consistent with the abnormally intense and prolonged inflammatory response that characterises the acute phase of this disease. In addition to proinflammatory cytokines, lipid mediators also play important roles in inflammation. Inflammatory lipid mediators derive mainly from the metabolism of polyunsaturated fatty acids (PUFAs). Consistently, our data demonstrate a relative increase of the polyunsaturated lipids in acute $\mathrm{CN}$ patients compared to controls. The key links between PUFAs and the inflammatory response are the eicosanoids. Eicosanoids are indeed involved in the modulation of both the intensity and the duration of inflammatory responses [26, 27]. Since inflammatory cells typically contain a high proportion of the n-6 PUFA arachidonic acid (20:4n-6) and low proportions of other 20-carbon PUFAs, arachidonic acid is usually the major substrate for eicosanoid synthesis during the inflammation process. However, detailed knowledge of tissue chemical composition and consequently the usage of appropriate 
standards are required to quantify absolute metabolite concentrations by MRS. Therefore, in the present study, fat content concentration changes in inflamed tissue were assessed by using the diallylic proton peak, which is detected exclusively in PUFAs, to calculate PUI, a lipid index that provides a measure of the relative concentration of the polyunsaturated lipids. PUI allows excellent measurement of the relative degree of polyunsaturation in oils [21] and would only be an imperfect technique for quantification in conjugated polyunsaturated fatty acids (PUFAs). However, conjugated PUFAs are in very low concentration in mammalian lipids [28]. In our study, we found a positive correlation between PUI baseline values and serum levels of IL- 6 and $\mathrm{TNF}-\alpha$, thus supporting the hypothesis that PUI could be a novel inflammatory marker in acute $\mathrm{CN}$.

After limb immobilisation, we observed a gradual reduction of PUI values in all patients. Most importantly, we found a negative correlation between the percentage reduction of PUI at 3 months and recovery time as assessed by bone marrow oedema disappearance as demonstrated on STIR images. As in our study the spectroscopic volume of interest (VOI) was positioned over the bone marrow oedema, the decrease in PUI observed during follow-up could have mirrored the decrease in bone marrow lesion volume/intensity. However, we did not detect any significant variations in the intensity or in the volume of the bone marrow oedema lesion at 3 months' follow-up (data not shown). Moreover, it should be noted that the healing time according to MRI was approximately 1 month longer than the PUI normalisation. Despite the fact that in follow-up acquisitions careful attention was paid to placing the voxel in exactly the same position as in baseline acquisitions, it cannot be excluded that even slight differences in voxel repositioning might have accounted for this discrepancy. However, this discrepancy could also be explained by the fact that the intensity and duration of bone marrow oedema may not necessarily go hand in hand with the intensity and duration of the underlying inflammatory process. Indeed, bone marrow oedema lesions on STIR images are not fully understood [29]. The increase in signal intensity on STIR has been attributed not only to increased permeability of small intramedullary vessels, but also to abnormal trabeculae, bone marrow necrosis or swelling of fat cells [29]. Thus, it is not inconceivable that despite the persistency of bone marrow oedema on MRI, MRS demonstrates the disappearance of metabolic alterations associated with the underlying inflammatory process.

Recently, it has been suggested that the presence of acute inflammation accompanying the onset of acute $\mathrm{CN}$ may directly or indirectly mediate the osteolysis observed in acute $\mathrm{CN}$ patients [30]. Also, there is evidence that marrow lipid composition can directly influence the quality of trabeculae [31]. Notably,
PGE2 is a potent stimulator of bone reabsorption and to date is the primary PG affecting bone metabolism [31]. In light of these considerations, we speculate that the increase in PUI that we observed in acute $\mathrm{CN}$ patients could be one of the multiple factors that may possibly contribute to the ongoing osteolysis in these patients. Along this line of reasoning, it has been reported that proinflammatory cytokines may play an important role in causing neuropathic osteoarthropathy [5].

A possible limitation of our study is that, despite the fact that in vivo MRS bone marrow quantitative lipid evaluation has been widely accepted, it may, however, overestimate the fraction of fat that is polyunsaturated [20]. Indeed, the in vivo determination of lipid levels and composition from proton MRS spectra obtained with clinically available MR systems raises several issues that may affect metabolite quantification. Macromolecules with chemical shifts overlapping the lipid resonances may alter the estimates of relative intensities. In addition, the signals in vivo are the sum of very complex 1-H spin-coupled multiplets from several different fatty acids with slightly different chemical shifts. Consequently, a single Lorentzian line, as used in our study, may not properly represent the observed line shape [32]. The usage of high-field MRI, such as 7-T systems, will certainly improve in vivo MRS quantification of lipid polyunsaturation levels.

In conclusion, our preliminary data provide a first step in applying MRS to the evaluation of bone marrow in patients with acute CN. Based on our data we suggest that MRS could be a complementary tool that can be employed to better understand the pathophysiology of acute $\mathrm{CN}$ and to obtain quantitative parameters that may help clinicians to monitor disease activity in patients with acute $\mathrm{CN}$. However, further studies are warranted to demonstrate whether the progressive normalisation of the PUI values observed under follow-up treatment actually corresponds to disease remission.

Acknowledgements We would like to give special thanks to Maurizio Ferretti for the very valuable technical assistance.

Marcello Cadioli is Clinical Scientist for Magnetic Resonance at Philips Medical Systems.

\section{References}

1. Nielson DL, Armstrong DG (2008) The natural history of Charcot's neuroarthropathy. Clin Podiatr Med Surg 25:53-62

2. Chantelau E (2005) The perils of procrastination: effects of early vs. delayed detection and treatment of incipient Charcot fracture. Diabet Med 22:1707-1712

3. Greenstein A, Jarrett SJ, McGonagle D (2005) Acute neuropathic joint disease: a medical emergency? Diabetes Care 28:2962-2964 
4. Uccioli L, Sinistro A, Almerighi C, Ciaprini C, Cavazza A, Giurato L et al (2010) Proinflammatory modulation of the surface and cytokine phenotype of monocytes in patients with acute Charcot foot. Diabetes Care 33:350-355

5. Jeffcoate W (2005) The role of proinflammatory cytokines in the cause of neuropathic osteoarthropathy (acute Charcot foot) in diabetes. Lancet 366:2058-2061

6. Sinacore DR, Withrington NC (1999) Recognition and management of acute neuropathic (Charcot) arthropathies of the foot and ankle. J Orthop Sports Phys Ther 29:736-746

7. Morrison WB, Ledermann HP (2002) Work-up of the diabetic foot. Radiol Clin North Am 40:1171-1192

8. Petrova NL, Moniz C, Elias DA, Buxton-Thomas M, Bates M, Edmonds ME (2007) Is there a systemic inflammatory response in the acute Charcot foot? Diabetes Care 30:997-998

9. Tomas MB, Patel M, Marwin SE, Palestro CJ (2000) The diabetic foot. Br J Radiol 73:443-50

10. Boyko EJ, Ahroni JH, Stensel VL (2001) Skin temperature in the neuropathic diabetic foot. J Diabetes Complications 15:260-4

11. Armstrong DG, Lavery LA (1996) Monitoring neuropathic ulcer healing with infrared dermal thermometry. J Foot Ankle Surg 35:335-8

12. Griffith J, Yeung D, Antonio G, Lee F, Hong A, Wong S et al (2005) Vertebral bone mineral density, marrow perfusion, and fat content in healthy men and men with osteoporosis: dynamic contrast-enhanced MR imaging and MR spectroscopy. Radiology 236:945-951

13. Griffith JF, Yeung DK, Chow SK, Leung JC, Leung PC (2009) Reproducibility of MR perfusion and (1)H spectroscopy of bone marrow. J Magn Reson Imaging 29:1438-42

14. Li X, Kuo D, Schafer AL, Porzig A, Link TM, Black D, Schwartz AV (2011) Quantification of vertebral bone marrow fat content using 3 Tesla MR spectroscopy: reproducibility, vertebral variation and applications in osteoporosis. J Magn Reson Imaging 33:974-979

15. Yeung D, Griffith J, Antonio G, Lee F, Woo J, Leung P (2005) Osteoporosis is associated with increased marrow fat content and decreased marrow fat unsaturation: a proton MR spectroscopy study. J Magn Reson Imaging 22:279-285

16. Li X, Ma BC, Bolbos RI, Stahl R, Lozano J, Zuo J et al (2008) Quantitative assessment of bone marrow edema-like lesion and overlying cartilage in knees with osteoarthritis and anterior cruciate ligament tear using MR imaging and spectroscopic imaging at 3 Tesla. J Magn Reson Imaging 28:453-61

17. Fedele D, Comi G, Coscelli C, Cucinotta D, Feldman EL, Ghirlanda G et al (1997) A multicenter study on the prevalence of diabetic neuropathy in Italy. Italian Diabetic Neuropathy Committee. Diabetes Care 20:836-843

18. Naressi A, Couturier C, Castang I, de Beer R, Graveron-Demilly D (2001) JAVA-based graphical user interface for MRUI, a software package for quantitation of in vivo/medical magnetic resonance spectroscopy signals. Comput Biol Med 31:269-286

19. Vanhamme L, van den Boogaart A, Van Huffel S (1997) Improved method for accurate and efficient quantification of MRS data with use of prior knowledge. J Magn Reson 129:35-43

20. Ren J, Dimitrov I, Sherry AD, Malloy CR (2008) Composition of adipose tissue and marrow fat in humans by $1 \mathrm{H}$ NMR at 7 Tesla. Journal of Lipid Research 49:2055-2062

21. Johnson NA, Walton DW, Sachinwalla T, Thompson CH, Smith K, Ruell PA et al (2008) Noninvasive assessment of hepatic lipid composition: advancing understanding and management of fatty liver disorders. Hepatology 47:1513-1523

22. Provencher SW (1993) Estimation of metabolite concentrations from localized in vivo proton NMR spectra. Magn Reson Med 30:672-679

23. Schlossbauer T, Mioc T, Sommerey S, Kessler SB, Reiser MF, Pfeifer KJ (2008) Magnetic resonance imaging in early stage Charcot arthropathy: correlation of imaging findings and clinical symptoms. Eur J Med Res 13:409-414

24. Zampa V, Bargellini I, Rizzo L, Turini F, Ortori S, Piaggesi A, Bartolozzi C (2011) Role of dynamic MRI in the follow-up of acute Charcot foot in patients with diabetes mellitus. Skeletal Radiol 40:991-9

25. Andersen PE (2012) Patient selection and preparation strategies for the use of contrast material in patients with chronic kidney disease World J Radiol 4:253-257

26. Lewis RA, Austen KF, Soberman RJ (1990) Leukotrienes and other products of the 5-lipoxygenase pathway: biochemistry and relation to pathobiology in human diseases. N Engl J Med 323:64555

27. Tilley SL, Coffman TM, Koller BH (2001) Mixed messages: modulation of inflammation and immune responses by prostaglandins and thromboxanes. J Clin Invest 108:15-23

28. Belury MA (2002) Dietary conjugated linoleic acid in health: physiological effects and mechanisms of action. Annu Rev Nutr 22:505-531

29. Zanetti M, Bruder E, Romero J, Hodler J (2000) Bone marrow edema pattern in osteoarthritic knees: correlation between MR imaging and histologic findings. Radiology 215:835-840

30. Sinacore DR, Hastings MK, Bohnert KL, Fielder FA, Villareal DT, Blair VP III et al (2008) Inflammatory osteolysis in diabetic neuropathic (Charcot) arthropathies of the foot. Phys Ther 88:1399 1407

31. Watkins BA, Li Y, Seifert MF (2001) Lipids as modulators of bone remodelling. Curr Opin Clin Nutr Metab Care 4:105-110

32. Knothe G, Kenar JA (2004) Determination of the fatty acid profile by H-1-NMR spectroscopy. Eur J Lipid Sci Tech 106:88-96 